\title{
Generating patient specific pseudo-CT of the head from MR using atlas-based regression
}

\author{
Jens Sjölund, Daniel Forsberg, Mats Andersson and Hans Knutsson
}

\section{Linköping University Post Print}

\section{Tweet}

N.B.: When citing this work, cite the original article.

Original Publication:

Jens Sjölund, Daniel Forsberg, Mats Andersson and Hans Knutsson, Generating patient specific pseudo-CT of the head from MR using atlas-based regression, 2015. Physics in Medicine and Biology, 60(2).

http://dx.doi.org/10.1088/0031-9155/60/2/825

Copyright: IOP Publishing: Hybrid Open Access

http://www.iop.org/

Postprint available at: Linköping University Electronic Press

http://urn.kb.se/resolve?urn=urn:nbn:se:liu:diva-113297 


\title{
Generating patient specific pseudo-CT of the head from MR using atlas-based regression
}

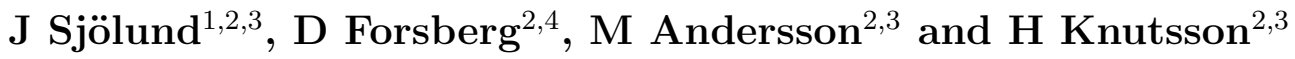 \\ ${ }^{1}$ Elekta Instrument AB, Kungstensgatan 18, Box 7593, SE-103 93 Stockholm, Sweden \\ ${ }^{2}$ Center for Medical Image Science and Visualization (CMIV), Linköping University, \\ Sweden \\ ${ }^{3}$ Department of Biomedical Engineering, Linköping University, Linköping, Sweden \\ ${ }^{4}$ Sectra, Teknikringen 20, SE-583 30, Linköping, Sweden \\ E-mail: jens.sjolund@liu.se
}

\begin{abstract}
Radiotherapy planning and attenuation correction of PET images require simulation of radiation transport. The necessary physical properties are typically derived from computed tomography (CT) images, but in some cases, including stereotactic neurosurgery and combined PET/MR imaging, only magnetic resonance (MR) images are available. With these applications in mind, we describe how a realistic, patient-specific, pseudo-CT of the head can be derived from anatomical MR images. We refer to the method as atlas-based regression, because of its similarity to atlas-based segmentation.

Given a target MR and an atlas database comprising MR and CT pairs, atlas-based regression works by registering each atlas MR to the target MR, applying the resulting displacement fields to the corresponding atlas CTs and, finally, fusing the deformed atlas CTs into a single pseudo-CT.

We use a deformable registration algorithm known as the Morphon and augment it with a certainty mask that allows a tailoring of the influence certain regions are allowed to have on the registration. Moreover, we propose a novel method of fusion, wherein the collection of deformed CTs is iteratively registered to their joint mean, and find that the resulting mean CT becomes more similar to the target CT. However, the voxelwise median provided even better results; at least as good as earlier work that required special MR imaging techniques. This makes atlas-based regression a good candidate for clinical use.
\end{abstract}

PACS numbers: 87.61.-c, 87.57.nj, 07.05.Pj

Keywords: Magnetic resonance imaging, Deformable registration, Computed Tomography, Radiotherapy, Attenuation correction, Morphon 


\section{Introduction}

Computed tomography (CT), with its high availability and geometric accuracy, has proven an invaluable tool for many clinical applications. Since a CT voxel's intensity relates to its attenuation of X-rays, CT images provide a means of estimating tissue electron density, which, in turn, is necessary to simulate radiation transport. For this reason, $\mathrm{CT}$ is a cornerstone of both radiotherapy planning and attenuation correction of Positron Emission Tomography (PET) images.

There are, however, at least two applications where electron density information is needed but only magnetic resonance (MR) images are readily available. The first application is in combined $\mathrm{PET} / \mathrm{MR}$ imaging - currently transitioning to reach its full clinical potential - where attenuation correction is necessary but CT acquisition would require an additional scan (Bezrukov, Mantlik, Schmidt, Schölkopf \& Pichler 2013). The second application is in certain types of radiotherapy planning, where CT's limited soft tissue contrast stunts accurate tumor delineation whereas MR provides valuable guidance. Such cases include stereotactic radiosurgery (Sjölund, Eriksson Järliden, Andersson, Knutsson \& Nordström 2014) of brain disorders and radiotherapy treatment of prostate cancer (Rasch, Barillot, Remeijer, Touw, van Herk \& Lebesque 1999).

A way of estimating electron densities in these applications is to model CT images, referred to as pseudo-CT images, based on the MR images. The benefits of this approach include reducing imaging costs, sparing the patient from X-ray exposure and eliminating registration errors between MR and CT images. A further incentive is the ongoing work on simultaneous radiotherapy and MR imaging (Raaymakers, Lagendijk, Overweg, Kok, Raaijmakers, Kerkhof, van der Put, Meijsing, Crijns, Benedosso et al. 2009), that brings the potential for truly adaptive, MR-based, treatment.

Several approaches of generating pseudo-CT images from MR images have been proposed. Perhaps the most straightforward is to manually segment a few tissue classes and assign different CT values to each class. This yields a clear improvement in dose calculation accuracy over the simplistic (but common) approximation that all tissue properties are equivalent to those of water (Jonsson, Karlsson, Karlsson \& Nyholm 2010). The clinical applicability is, however, limited as long as a time-consuming manual segmentation is required; an automatic method is evidently needed.

Automatic methods of mapping MR images to electron density data can broadly be categorized into atlas-based and data-based approaches.

The atlas-based methods typically employ deformable registration to match a reference dataset to a new patient. Either one can directly register an atlas CT to the target MR image (Schreibmann, Nye, Schuster, Martin, Votaw \& Fox 2010) or one can relate them through an atlas MR that has previously been registered to a CT (Dowling, Lambert, Parker, Salvado, Fripp, Capp, Wratten, Denham \& Greer 2012). When the latter approach is used for segmentation it is often referred to as atlas-based segmentation (Stanescu, Jans, Pervez, Stavrev \& Fallone 2008). Because atlas-based methods rely heavily on prior information, they are more robust, and are therefore better 
equipped to deal with image artifacts such as those caused by metal implants. This robustness is, regrettably, a double-edged sword, since it may be unable to account for patients with atypical anatomy. If a single anatomy is used the (often arbitrary) choice of atlas will cause a bias in the output and makes it particularly sensitive to topological differences in the anatomy. A way of mitigating this is to use a database of atlas patients that are separately registered. Just as in any estimation procedure the use of several independent measurements allows a more accurate estimate, which has been verified in several studies (Heckemann, Hajnal, Aljabar, Rueckert \& Hammers 2006, Burgos, Cardoso, Modat, Pedemonte, Dickson, Barnes, Duncan, Atkinson, Arridge, Hutton et al. 2013, Uh, Merchant, Li, Li \& Hua 2014). Both how to select the atlas patients (Aljabar, Heckemann, Hammers, Hajnal \& Rueckert 2009) and how to fuse the segmentations (Artaechevarria, Munoz-Barrutia \& Ortiz-de-Solorzano 2009) are areas that deserve additional attention.

Data-based approaches directly associate intensities in the MR images with the Hounsfield units of a CT, either by segmentation and subsequent density assignment (Hsu, Cao, Huang, Feng \& Balter 2013, Sjölund et al. 2014) or by direct regression (Kapanen \& Tenhunen 2013). This is complicated by the fact that MR intensitiesunlike CT - are not calibrated and some type of intensity normalization, also known as bias-field correction, is often needed as a preprocessing step. Another difficulty is that, due to the extremely short $\mathrm{T} 2$ relaxation time of bone, neither bone nor air provide any signal in anatomical MR and are therefore indistinguishable with respect to intensity values. This issue can be tackled by using an MR sequence with ultrashort echo time (UTE), such that a signal from bone is acquired (Johansson, Karlsson \& Nyholm 2011, Johansson, Garpebring, Karlsson, Asklund \& Nyholm 2013). However, this requires additional - valuable - scanner time.

Hybrid methods are also conceivable, e.g. Hofmann et al (Hofmann, Steinke, Scheel, Charpiat, Farquhar, Aschoff, Brady, Schölkopf \& Pichler 2008) used a database of registered MR-CT pairs, which were registered to the patient in a classical atlas approach. For each voxel in the patient image, a pseudo-CT value based on the spatially closest voxels from the images in the atlas database was predicted using Gaussian process regression. However, a recent study (Uh et al. 2014), found that this post-processing step did not yield an improved performance compared with the arithmetic mean.

This work corroborates the finding that a simple regression method is hard to improve upon. We employ a multi-atlas based method, using a deformable registration algorithm particularly suited for registering images with varying contrast (such as MR) (Knutsson \& Andersson 2005). We propose a novel fusion method whereby the deformed CTs are iteratively registered to their joint mean and show that this makes the resulting mean CT more similar to the real CT. However, the voxelwise median remains a significantly better estimator, with a performance at least as good as previously reported. 


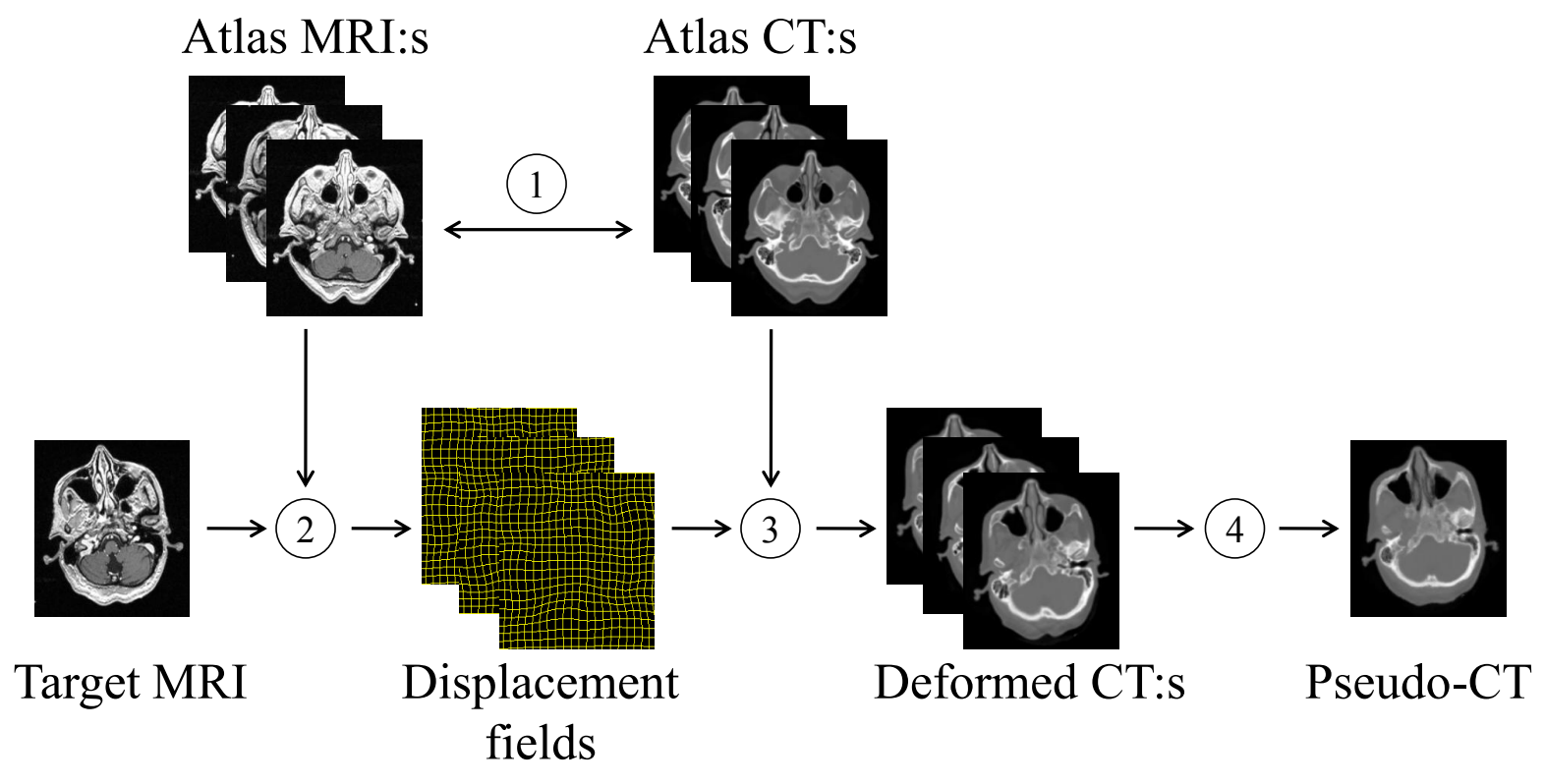

Figure 1: Outline of the atlas-based regression algorithm: (1) MR and CT pairs are collected and rigidly registered, (2) each atlas MR is registered to the target MR, (3) the resulting transformation is applied to the corresponding CTs and (4) the set of deformed CTs are fused to a single pseudo-CT.

\section{Method}

Because of the similarity with atlas-based segmentation, we refer to the framework of our method as atlas-based regression. It consists of four general steps, outlined in figure 1 .

(i) Collect an atlas database comprising MR and CT pairs.

(ii) Register each atlas MR to the target MR.

(iii) Apply the same transformation to the corresponding atlas CT.

(iv) Fuse the collection of deformed CTs into a single pseudo-CT.

In the following, each of these steps is detailed, after which the performance measure, used in the subsequent evaluation, is described.

\subsection{Atlas creation}

Ten patients were chosen at random from a collection of patients having previously undergone stereotactic radiosurgery using the Leksell Gamma Knife ${ }^{\circledR}$. The only constraint guiding the selection was that the studies contained both an MR and a CT where the whole head was in the field-of-view. The MR images were acquired with a 1.5 T Siemens Avanto using a T1-weighted 3D spoiled gradient recalled echo sequence (echo time $4.6 \mathrm{~ms}$, repetition time $11 \mathrm{~ms}$, flip angle $20^{\circ}$, voxel size $1 \mathrm{x} 1 \mathrm{x} 1 \mathrm{~mm}^{3}$ ) without contrast agent. As will be discussed further, the registration method used is not 

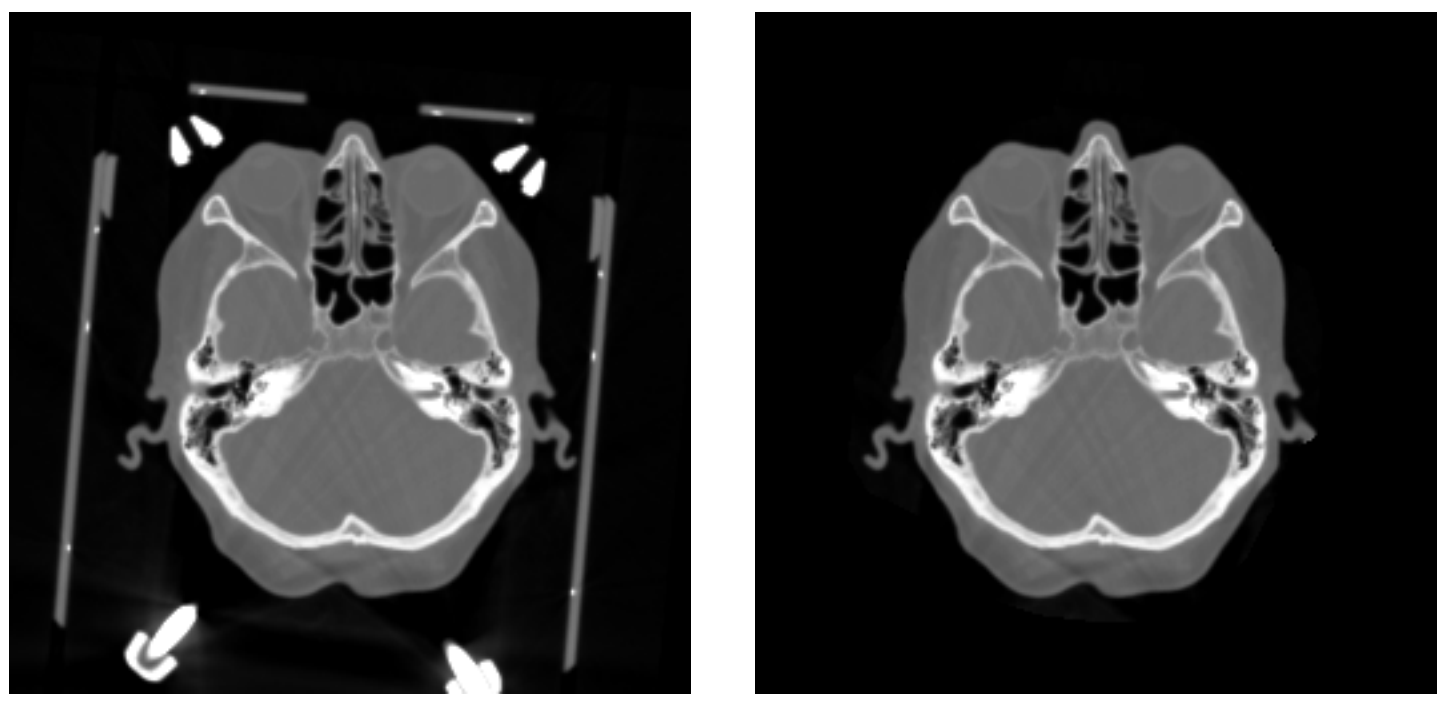

Figure 2: The stereotactic frame was manually removed in the atlas CTs.

sensitive to the particular choice of sequence - only that it is the same for the images being registered. The corresponding CT images were acquired with a Siemens Sensation 16 with tube voltage $120 \mathrm{kV}$, exposure $300 \mathrm{mAs}$, in-plane resolution $0.5 \mathrm{x} 0.5 \mathrm{~mm}^{2}$ and slice thickness $1 \mathrm{~mm}$.

The stereotactic frame that is often used in conjunction with GammaKnife treatments is equipped with fiducials visible in both MR and CT. These were used to rigidly register and resample the $\mathrm{CT}$ to the $\mathrm{MR}$ images using the accompanying software, Leksell GammaPlan ${ }^{\circledR}$.

However, this frame - being positioned differently on each patient - is clearly visible on the CT images and in order not to corrupt the pseudo-CT, the atlas CTs were manually masked such that most of the stereotactic frame was removed, see figure 2. This only needs to be done for the atlas patients, and since the atlas construction is presumably done just once, it would not be of any concern if used clinically.

Moreover, since the studies are retrospective, abnormalities in the anatomy is the rule rather than the exception.

\subsection{Deformable image registration}

We use deformable image registration to find displacement fields that attempt to map the atlas MRs to the target MR. For each voxel, a displacement vector is estimated that points to the best corresponding location in another image, while respecting certain constraints on the resulting deformation. For example, an anatomically realistic displacement field must be diffeomorphic (Arsigny, Commowick, Pennec \& Ayache 2006, Janssens, Jacques, de Xivry, Geets \& Macq 2011).

Denote the displacement field $\boldsymbol{d}$, the source image (an atlas MR) $\mathcal{I}_{S}$ and the target image (the target MR) $\mathcal{I}_{\mathrm{T}}$. The objective is, thus, to find an anatomically realistic $\boldsymbol{d}$ 
that optimizes

$$
\mathcal{M}\left(\mathcal{I}_{S}\left(\boldsymbol{x}+\boldsymbol{d}(\boldsymbol{x}), \mathcal{I}_{\mathrm{T}}(\boldsymbol{x})\right)\right)
$$

where $\mathcal{M}$ is a measure of image similarity and $\boldsymbol{x}$ is the set of spatial coordinates. We will henceforth refer to $\mathcal{I}_{S}(\boldsymbol{x}+\boldsymbol{d}(\boldsymbol{x}))$ as the deformed image and denote it $\mathcal{I}_{\mathrm{D}}(\boldsymbol{x})$.

Much of the research on deformable image registration for medical applications has focused on CT images, which - as mentioned before - have intensity values that are calibrated. To use an algorithm with an intensity-based similarity measure, such as the popular demons algorithm (Thirion 1998), for MR to MR registration one must first apply a bias-field correction (Sled, Zijdenbos \& Evans 1998); a step which is time-consuming in itself and introduces an additional uncertainty. One could instead use an information-theoretic measure of image similarity, such as (normalized) mutual information (Viola \& Wells III 1997, Pluim, Maintz \& Viergever 2003), which is commonly used for intermodality registration. This comes at the cost of increased computational complexity.

Another option is to use the Morphon (Knutsson \& Andersson 2005), a registration algorithm that matches transitions between dark and bright regions rather than intensities. It is based on the local phase of spatial variations of intensities and is therefore invariant to intensity mean and scaling, making it suitable for registering images with varying contrast.

The local phase is estimated by convolving the image with quadrature filters, a type of complex wavelets (Knutsson \& Andersson 2003), tuned to a certain frequency and direction. In 3D, six directions $\hat{\boldsymbol{n}}_{k}$ with accompanying filters $f_{k}$ are required (Knutsson 1989) $(k=1, \ldots, 6)$. Let the result of convolving the deformed and target images with quadrature filter $f_{k}$ be denoted $Q_{\mathrm{D}_{k}}$ and $Q_{T_{k}}$, respectively, i.e.

$$
\begin{aligned}
& Q_{\mathrm{D}_{k}}=\mathcal{I}_{\mathrm{D}} * f_{k}, \\
& Q_{\mathrm{T}_{k}}=\mathcal{I}_{\mathrm{T}} * f_{k} .
\end{aligned}
$$

The local phase estimates, $\phi_{\mathrm{D}_{k}}$ and $\phi_{\mathrm{T}_{k}}$, for each filter direction are the arguments of the corresponding complex filter responses. The local phase difference $\Delta \phi_{k}$ is thus

$$
\Delta \phi_{k}=\arg \left(Q_{\mathrm{D}_{k}} Q_{\mathrm{T}_{k}}^{*}\right)
$$

where the star denotes complex conjugation. The Morphon assigns different weights on the importance of matching in different regions and orientations using a certainty $c_{k}$ derived from the local amplitude and phase estimates as

$$
c_{k}=\left|Q_{\mathrm{D}_{k}} Q_{\mathrm{T}_{k}}^{*}\right|^{1 / 2} \cos ^{2}\left(\frac{\Delta \phi_{k}}{2}\right) .
$$

The filter responses $Q_{\mathrm{T}_{k}}$ are also used to compute the local structure tensor $T$ of the deformed image as

$$
T=\sum_{k=1}^{6}\left|Q_{\mathrm{T}_{k}}\right|\left(\hat{\boldsymbol{n}}_{k} \hat{\boldsymbol{n}}_{k}^{T}-\frac{1}{5} I\right)
$$

where $I$ is the identity matrix. 
The Morphon uses the local phase differences between signals of similar local frequency to estimate the spatial shift between them. Given that images can locally be considered as narrow band signals, the computation of the local phase difference is equivalent to the estimation of the local displacement between images (Knutsson \& Andersson 2003). Mathematically-using a subscript $i$ to refer to the variable in question evaluated at coordinate $\boldsymbol{x}_{i}$ - the displacement estimate $\boldsymbol{d}_{i}$ is found by solving the weighted least squares problem

$$
\begin{aligned}
& \boldsymbol{d}_{i}=\underset{\boldsymbol{d}_{i}}{\operatorname{argmin}} \sum_{k=1}^{6}\left\|c_{k, i} T_{i}\left(\boldsymbol{d}_{i}-\Delta \phi_{k, i} \boldsymbol{n}_{k}\right)\right\|_{2}^{2} \\
& =\underset{\boldsymbol{d}_{i}}{\operatorname{argmin}}\left\|\left(\begin{array}{ccc}
c_{1, i} T_{i} & & \\
& \ddots & \\
& & c_{6, i} T_{i}
\end{array}\right)\left(\mathbb{1}_{18 \times 3} \boldsymbol{d}_{i}-\left(\begin{array}{c}
\Delta \phi_{1} \hat{\boldsymbol{n}}_{1} \\
\vdots \\
\Delta \phi_{6} \hat{\boldsymbol{n}}_{6}
\end{array}\right)\right)\right\|_{2} \\
& \stackrel{\text { def }}{=} \underset{\boldsymbol{d}_{i}}{\operatorname{argmin}}\left\|W\left(A \boldsymbol{d}_{i}-b\right)\right\|_{2} \\
& =\left(A^{T} W^{T} W A\right)^{-1} A^{T} W^{T} W b
\end{aligned}
$$

where columns vectors are assumed and $\mathbb{1}$ is a matrix of all ones (with dimension indicated by the subscript).

Just as most other deformable registration algorithms, the Morphon is an iterative algorithm. At each iteration, the estimation of the displacement field $\boldsymbol{d}$ is followed by a field accumulation, wherein $\boldsymbol{d}$ is combined with the previously accumulated displacement field $\boldsymbol{d}_{\mathrm{a}}$. The field accumulation is performed with the diffeomorphic update formula (Janssens et al. 2011)

$$
\boldsymbol{d}_{\mathrm{a}} \leftarrow \boldsymbol{d}_{\mathrm{a}} \circ \exp (\boldsymbol{d})
$$

where $\circ$ denotes compositive accumulation. The exponential map is approximated by a scaling and squaring procedure (Higham 2005).

To make the assumption of narrow band signals valid, the registration is performed over multiple scales, starting on coarser scales to register large global displacements and moving on to finer scales to register smaller local deformations. To further increase robustness, the displacement field is constrained to be affine in the first iterations and the subsequent deformable steps employ both fluid regularization (low pass filtering of $\boldsymbol{d}$ ) and elastic regularization (low pass filtering of $\boldsymbol{d}_{\mathrm{a}}$ ).

The implementation of the Morphon that we have used, freely available for download $\ddagger$ takes advantage of the massive parallelism of the graphics card (GPU) to drastically reduce the computation time (Forsberg, Eklund, Andersson \& Knutsson 2011). As a consequence the phase-based deformable registration takes less than a minute. An earlier implementation is part of the Insight Segmentation and Registration Toolkit (ITK) (Plumat, Andersson, Janssens, Orban de Xivry, Knutsson \& Macq 2009). $\ddagger$ https://bitbucket.org/dforsberg 

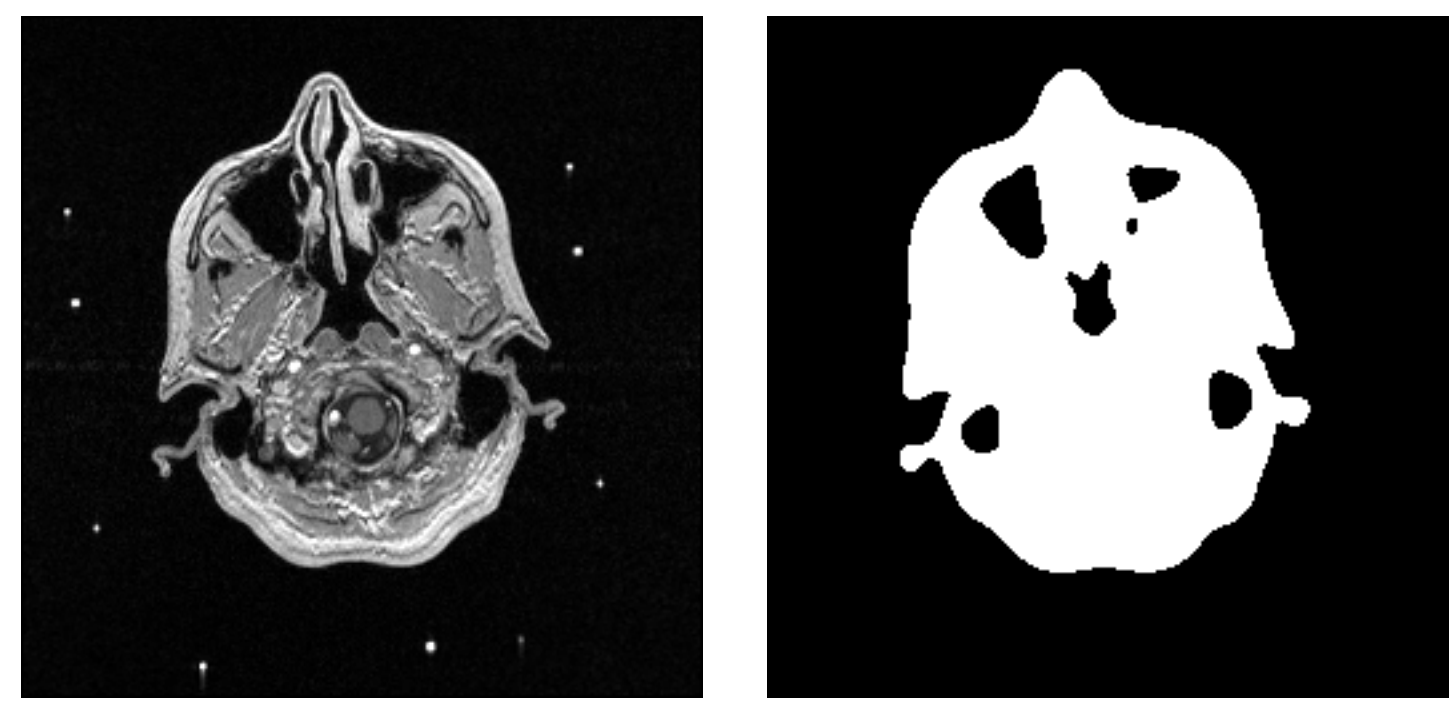

Figure 3: To reduce the impact of fiducials and image artefacts, the registration only estimates the displacement field based on voxels contained in a certainty mask (right). In this work, certainty masks were derived by low-pass filtering the MRs and thresholding the resulting images using Otsu's method.

To exclude that the fiducials and/or noise and image artifacts in the surrounding air adversely affect the registration, we augment the default certainty measure $c_{k}$ with an additional certainty map that multiplies the existing certainty map in (5). Although we have used a binary mask, derived by applying a 15x15x15 mm³ Gaussian low-pass filter and thresholding using Otsu's method (Otsu 1979), one could with this method fine tune the influence certain regions are allowed to have on the registration. For example, one might wish to put less emphasis on the nasal cavity where the anatomical variation is known to be large, or to disregard a region where a metal implant corrupts the MR signal.

\subsection{Forming a pseudo-CT from deformed CTs}

The deformable registration results in a set of displacement fields $\left\{\boldsymbol{d}^{(l)} \mid l=1, \ldots, L\right\}$, where the superscript denotes the atlas patient's index, that approximately optimize

$$
\mathcal{M}\left(\mathrm{MR}_{\mathrm{D}}^{(l)}, \mathrm{MR}_{\mathrm{T}}\right), \quad l=1, \ldots, L .
$$

By the assumption underpinning atlas-based registration methods, it follows that

$$
\mathcal{M}\left(\mathrm{CT}_{\mathrm{D}}^{(l)}, \mathrm{CT}_{\mathrm{T}}\right), \quad l=1, \ldots, L
$$

is also approximately optimized. The fusion step consolidates the set of deformed CTs into a single pseudo-CT, denoted $\widetilde{\mathrm{CT}}_{\mathrm{T}}$. In other words, there is a fusion function, $F$, such that

$$
F\left(\mathrm{CT}_{\mathrm{D}}^{(1)}, \ldots, \mathrm{CT}_{\mathrm{D}}^{(L)}\right)=\widetilde{\mathrm{CT}}_{\mathrm{T}}
$$


In the case of multi-atlas segmentation, where the deformed data is labeled, a wide range of so called label fusion approaches have been suggested (Artaechevarria et al. 2009). Even though their performance depends on intensity characteristics of the segmented region - local methods outperform global when there is high contrast and vice versa - assigning the label that the most segmentations agree upon, referred to as majority voting, remains the most common method, both because of its simplicity and its effectiveness (Rohlfing, Russakoff \& Maurer Jr 2004).

In the continuous case, majority voting (without modification) is doomed to fail simply because it is highly unlikely that any two outputs agree perfectly. One might argue that the most natural generalization of majority voting would be the (voxelwise) median over candidates,

$$
\widetilde{\mathrm{CT}}\left(\boldsymbol{x}_{i}\right)=\operatorname{median}\left(\mathrm{CT}_{\mathrm{D}}^{(1)}\left(\boldsymbol{x}_{i}\right), \ldots, \mathrm{CT}_{\mathrm{D}}^{(L)}\left(\boldsymbol{x}_{i}\right)\right)
$$

A major advantage of the median compared to the mean is its robustness to outliers and the ability to better deal with multimodal distributions - when there is an odd number of samples the median falls onto one of the sample values and does not risk ending up in between modes.

Before applying any estimator, however, we propose to improve the consistency of the deformed CTs by iteratively forming their (voxelwise) mean, registering each deformed CT to the mean, forming a new mean estimate and so forth. In pseudocode:

$$
\begin{gathered}
\text { for } j=1 \text { to numIterations do } \\
\mathcal{I}_{\mathrm{T}} \leftarrow \operatorname{mean}\left(\mathrm{CT}_{\mathrm{D}}^{(1)}, \ldots, \mathrm{CT}_{\mathrm{D}}^{(L)}\right) \\
\text { for } l=1 \text { to } L \text { do } \\
\mathcal{I}_{\mathrm{S}} \leftarrow \mathrm{CT}_{\mathrm{D}}^{(l)} \\
\boldsymbol{d}=\operatorname{Morphon}\left(\mathcal{I}_{\mathrm{S}}, \mathcal{I}_{\mathrm{T}}\right) \\
\mathrm{CT}_{\mathrm{D}}^{(l)}(\boldsymbol{x}) \leftarrow \mathcal{I}_{\mathrm{S}}(\boldsymbol{x}+\boldsymbol{d})
\end{gathered}
$$

end for

\section{end for}

return $F\left(\mathrm{CT}_{\mathrm{D}}^{(1)}, \ldots, \mathrm{CT}_{\mathrm{D}}^{(L)}\right)$

In principle, the procedure can be understood as first forming a CT estimate by taking the voxelwise mean. Quite likely, this estimate will be outside the space of feasible deformations of each of the atlas CTs. Therefore, we construct its projection onto each of these spaces by a registration and again form the voxelwise mean - which is likely to be closer to a feasible deformation than the previous - and repeat the procedure.

\subsection{Evaluation}

As opposed to MR images, the Hounsfield values of CT images are quantitative (for a monoenergetic spectrum). For this reason, one can quantify the error of the pseudo-CT by comparing it voxelwise to the target $\mathrm{CT}$ and summarizing it as for example the mean absolute error $\mathcal{E}$, where the mean is taken over the entire field-of-view (FOV),

$$
\mathcal{E}=\frac{1}{N_{\mathrm{FOV}}} \sum_{i \in \mathrm{FOV}}\left|\widetilde{\mathrm{CT}}_{\mathrm{T}}\left(\boldsymbol{x}_{i}\right)-\mathrm{CT}_{\mathrm{T}}\left(\boldsymbol{x}_{i}\right)\right|,
$$



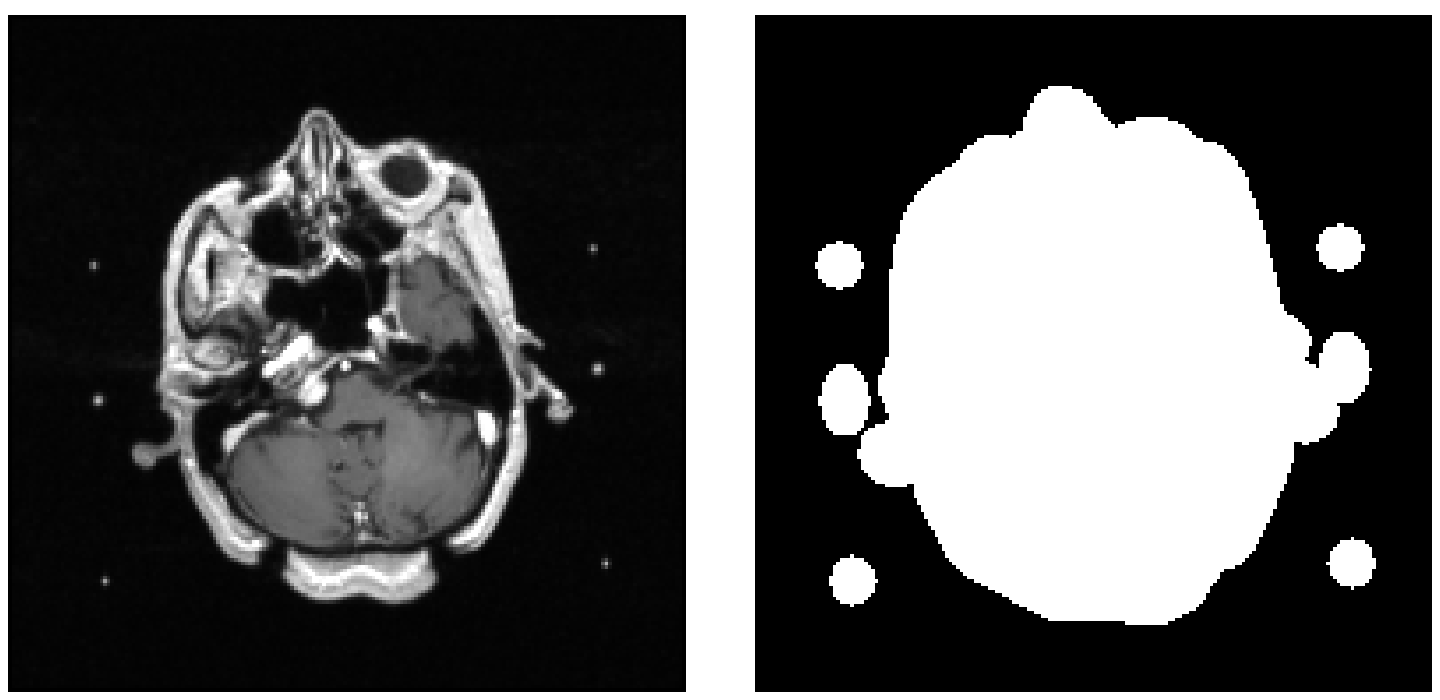

Figure 4: To make the error measures independent of the amount of air in the field of view, they were only computed for voxels in a mask supposed to correspond to the imaged subject. The mask was generated in a way identical to a previous study in order to allow comparison of the results. Another possibility would be to use the external contour of the patient.

where $N_{\text {FOV }}$ is the number of voxels in the field-of-view. We argue that this may lead to an unfair comparison in cases where much of the (easily classified) surrounding air is included in the averaging. To counteract this, we instead average the absolute error over a binary mask corresponding to the region of interest. To allow a comparison with the method of Johansson et al (Johansson et al. 2013), all error measures were computed on images downsampled to have voxel size $1.33 \times 1.33 \times 1.33 \mathrm{~mm}^{3}$. Also, the binary mask was derived in the same way: first the target MR is segmented into high and low intensity parts using Otsu thresholding (Otsu 1979); then the low intensity part is morphologically eroded using a spherical kernel with a diameter of 13 voxels; the largest connected component is classified as air and the largest connected component of the non-air is classified as the imaged subject. An example of a slice of the resulting mask is shown in figure 4 .

It is easier to obtain a good result for an image with low resolution because it is less sensitive to spatial differences. On the other hand, one might argue that, far from the focus of radiation, spatial offsets between the actual and modeled CT are of minor importance for the intended applications. To capture this, we propose to adapt an error measure - the $\gamma$-index - commonly used to compare dose distributions when performing quality assurance in radiotherapy (Low, Harms, Mutic \& Purdy 1998). The $\gamma$-index puts spatial deviations and dose deviations on equal footing by scaling them according to what deviations are acceptable in each domain separately. More specifically, we assume that we can tolerate a deviation of $\Delta H=100 \mathrm{HU}$ and a (spatial) distance to 
agreement of $\Delta x=2 \mathrm{~mm}$. The $\gamma$-index at the coordinate $\boldsymbol{x}_{i}$ is then calculated as

$$
\gamma\left(\boldsymbol{x}_{i}\right)=\min _{\boldsymbol{x}} \sqrt{\left(\frac{H_{\mathrm{ref}}\left(\boldsymbol{x}_{i}\right)-H_{\mathrm{est}}(\boldsymbol{x})}{\Delta H}\right)^{2}+\left(\frac{\left\|\boldsymbol{x}_{i}-\boldsymbol{x}\right\|}{\Delta x}\right)^{2}}
$$

where $H(\boldsymbol{x})$ is the Hounsfield unit at coordinate $\boldsymbol{x}$ and the subscripts "ref" and "est" refers to the reference volume and the estimated volume, respectively. Intuitively, this means that the $\gamma$-index is the closest distance from the point $\boldsymbol{x}_{i}$ on the reference surface (here 4D) to the estimated surface, a reasoning not unlike total least-squares. If $\gamma\left(\boldsymbol{x}_{i}\right) \leq 1$ the calculation at $\boldsymbol{x}_{i}$ passes, otherwise it fails.

We used a freely available C++-implementation§ (Persoon, Podesta, van Elmpt, Nijsten \& Verhaegen 2011) to compute the $\gamma$-index with the maximum search distance set to $2 \cdot \Delta x=4 \mathrm{~mm}$.

\section{Results}

We used leave-one-out cross-validation to evaluate a pseudo-CT generated for each of the ten patients. Both the affine and the deformable registration was done on three scales: with resolution downsampled 2, 4 and 8 times. The affine registration was done using 8, 10 and 15 iterations on the respective scales and the subsequent deformable registration was done using 1, 5, and 10 iterations. The fluid regularization consisted of convolving the estimated displacement field $\boldsymbol{d}$ with a Gaussian kernel with standard-deviation 3 voxels, the same on each scale. Similarly, the elastic regularization consisted of convolving the accumulated displacement field $\boldsymbol{d}_{\mathrm{a}}$ with a Gaussian kernel with standard-deviation 1.5 voxels. These parameters were found to be appropriate for the MR sequence at hand; a different sequence might require different parameters.

The iterated registration to the mean was done using one affine step followed by three deformable registration steps, wherein the mean was recomputed after each. We compared different ways of fusing the results: voxelwise mean and median (before and after iterated registration to mean) as well as the single best deformed CT (i.e. with the smallest mean absolute error compared to the ground truth). Table 1 summarizes the performance of the different estimators as measured by the mean absolute error, where the mean is computed over the binary mask described in section 2.4 and illustrated in figure 4. Using these values we found that the median, before iterated registration to mean, was a significantly better estimator than the mean after iterated registration $(p=0.0098)$ and the single best deformed CT $(p=0.0029)$ according to a sided Wilcoxon signed-rank test. More precisely, it was possible to reject the null hypothesis, that the matched estimates come from distributions whose medians are the same, in favor of the alternative hypothesis, that the error of the median-estimator is smaller, with a probability of error given by $p$ and the standard level of significance $p=0.05$. Figure 5 gives a visual comparison of the different estimators.

$\S$ http://www.mistir.info/ 
Generating pseudo-CT from MR using atlas-based regression

Table 1: Mean absolute errors (HU) for voxels in the evaluation mask.

\begin{tabular}{lcllll}
\hline Patient & Mean & Iterated mean & Median & Iterated median & Single best \\
\hline 1 & 138.6 & 131.8 & $\mathbf{1 2 5 . 9}$ & 126.7 & 127.7 \\
2 & 125.0 & 123.0 & $\mathbf{1 2 2 . 6}$ & 122.7 & 139.8 \\
3 & 117.8 & 114.5 & $\mathbf{1 0 5 . 8}$ & 113.9 & 113.2 \\
4 & 96.9 & 90.9 & 89.2 & $\mathbf{8 7 . 3}$ & 106.6 \\
5 & 109.5 & 101.2 & 98.6 & $\mathbf{9 6 . 7}$ & 112.5 \\
6 & 140.0 & 131.1 & $\mathbf{1 2 1 . 6}$ & 125.8 & 142.4 \\
7 & 161.0 & 161.7 & 149.9 & 158.6 & $\mathbf{1 4 4 . 2}$ \\
8 & 110.4 & 101.1 & 96.8 & $\mathbf{9 6 . 5}$ & 121.4 \\
9 & 115.4 & 109.5 & 113.6 & $\mathbf{1 0 3 . 8}$ & 130.1 \\
10 & 116.8 & 114.2 & $\mathbf{1 1 0 . 1}$ & 113.5 & 118.2 \\
\hline Mean & $123.2 \pm 18.6$ & $117.9 \pm 20.3$ & $113.4 \pm 17.6$ & $114.5 \pm 20.5$ & $125.6 \pm 13.4$ \\
\hline
\end{tabular}

We also found that the iterated registration to the mean made a significant improvement to the mean-estimator $(p=0.0020)$, as exemplified by figure $5 \mathrm{c}$ and $5 \mathrm{~d}$. The effect on the median was not statistically significant, although the median before iterated registration had the smallest mean absolute error: $113.4 \pm 17.6 \mathrm{HU}$. In a study using an MR sequence with ultrashort echo time together with Gaussian mixture regression (Johansson et al. 2013), the corresponding error was $130 \pm 18$ HU. In another method combining registration and pattern recognition (Hofmann et al. 2008), a mean absolute error of $100.7 \mathrm{HU}$ was reported. Resampling the results of the proposed method to the resolution and field of view used in that study reduced the mean absolute error to $60.5 \pm 9.4 \mathrm{HU}$.

Figure 6 shows the target $\mathrm{CT}$ and the pseudo-CT together with an image of the resulting $\gamma$-indices for the patients with the best (patient 4), average (patient 9) and worst (patient 7) performance with respect to the mean absolute error of the pseudoCT derived using the median. The largest errors occur at bone-tissue and air-tissue interfaces and in regions with a large anatomical variability, for example in the paranasal sinuses (cf. figure 6f).

\section{Discussion}

The Morphon proved highly robust for MR-MR registration, and as an unexpected side effect there was not as great need for the iterated registration to mean as anticipated. Nevertheless, the performance when using the median appears at least as good as that of a study requiring special MR imaging (Johansson et al. 2013). The two methods should, however, be evaluated on the same data before drawing any decisive conclusions.

A multitude of fusion strategies for atlas-based segmentation have been proposed, many of which can be expressed in the form of a general weighted voting (Artaechevarria et al. 2009), and a possible future research direction would be to investigate whether 


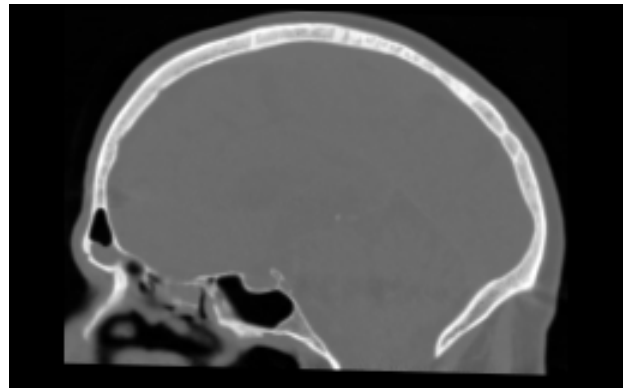

(a)

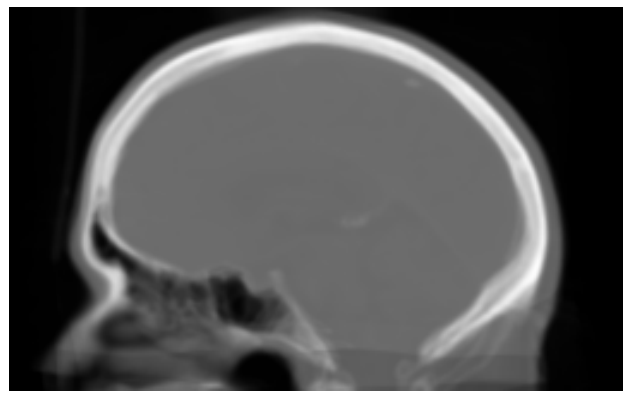

(c)

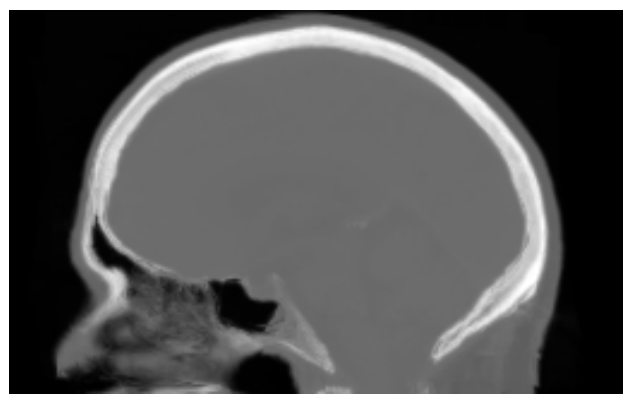

(e)

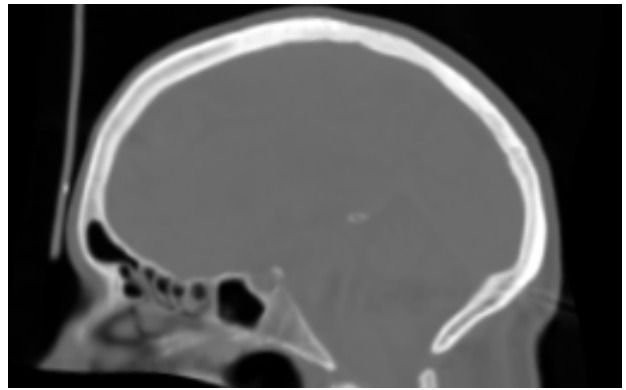

(b)

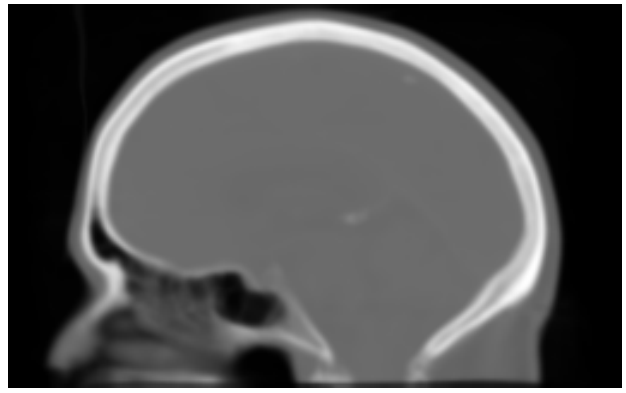

(d)

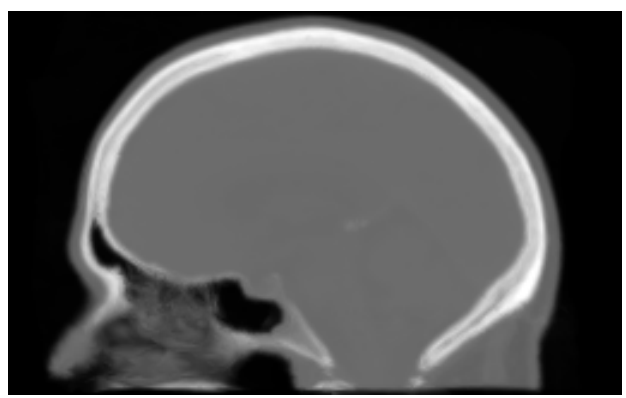

(f)

Figure 5: Pseudo-CTs for patient 9 (that had the average performance with respect to the mean absolute error of the pseudo-CT derived using the median): (a) ground truth (b) single best deformed CT (c) mean before iterated registration to mean (d) mean after iterated registration to mean (e) median before iterated registration to mean (f) median after iterated registration to mean.

some of these can be adapted to yield additional improvements. Since the registrations are carried out independently, any reasonable fusion function should be invariant to permutations of the indexes $l$.

Despite even the best efforts at combining the registered images, there remains a possibility of a systematic error either because the anatomy of the query subject is not, or not sufficiently, represented by the atlas database or because the degrees of freedom of the registration algorithm fail to adequately provide perfect small-scale alignment. On the upside, atlas-based regression may, for the same reasons, (partially) remove artifacts 


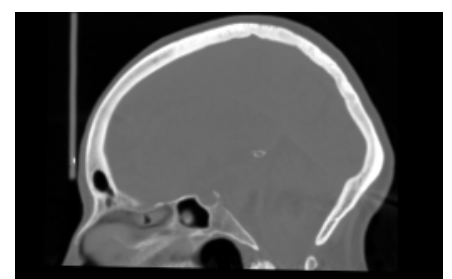

(a)

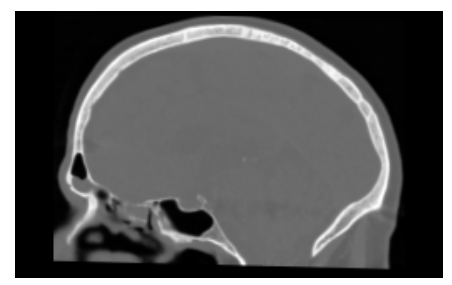

(d)

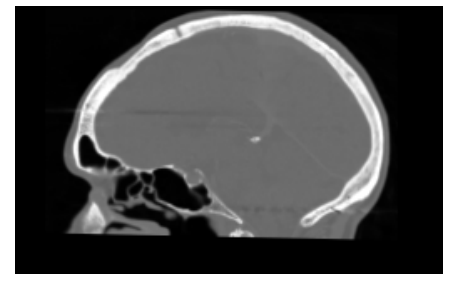

(g)

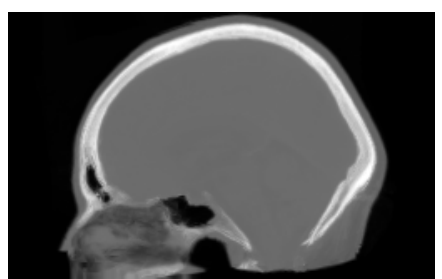

(b)

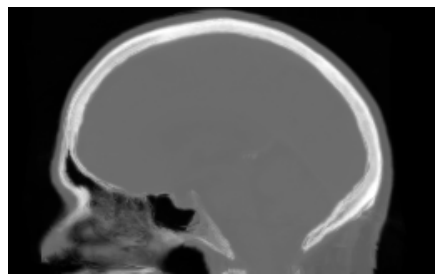

(e)

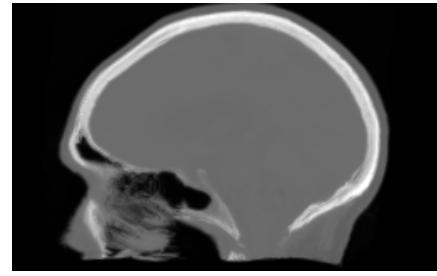

(h)

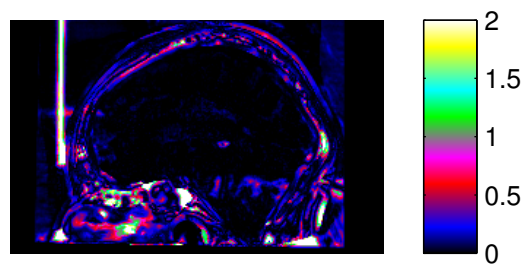

(c)

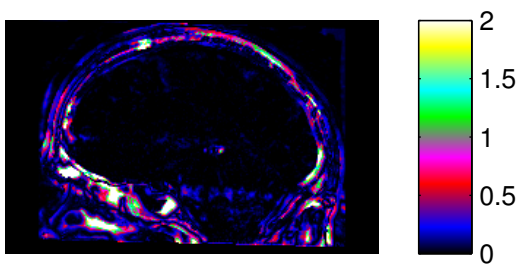

(f)

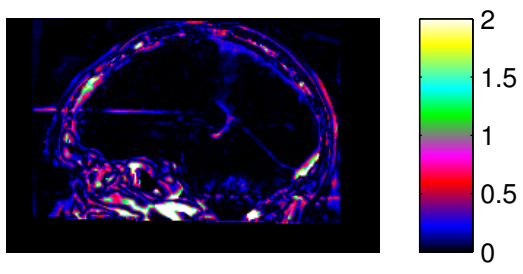

(i)

Figure 6: Target CTs (left column), pseudo-CTs derived using the median (center column) and the corresponding $\gamma$-index volumes (right column). From top to bottom, the rows correspond to the patients with the best (patient 4), average (patient 9) and worst (patient 7) performance with respect to the mean absolute error of the pseudo-CT derived using the median.

like beam hardening, as evident from figure 7. Incidentally, the type of artifacts shown in figure 7 were present in all the patients in this study.

Possibly, one could use the standard deviation of estimates to identify regions requiring further analysis. This could be done using a machine learning algorithm that also takes the MR, or some features derived thereof, as input (Hofmann et al. 2008, Sjölund et al. 2014).

A question of practical importance is how many atlases that need to be considered given the inter-patient variability of the anatomy in question. As more segmentations are fused, overlap accuracy increases because random errors, associated with individual registrations and atlas variability, are increasingly canceled out. However, the computational cost increases linearly with the number of patients and at some point the increase in accuracy is not motivated when weighted against time constraints. Provided that a large set of candidate atlases is available, the choice of which to include as atlas patients is pertinent. A recent study (Aljabar et al. 2009) on atlas-based segmentation 


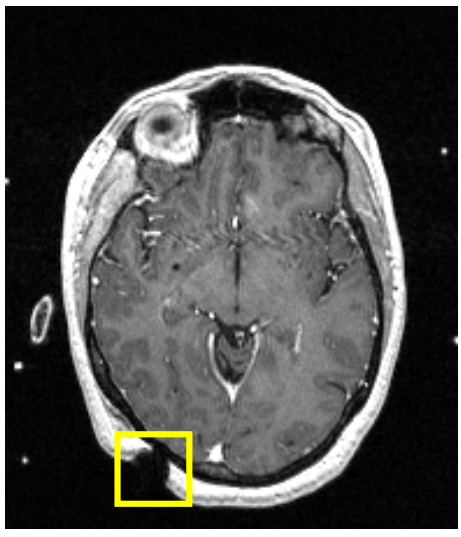

(a)

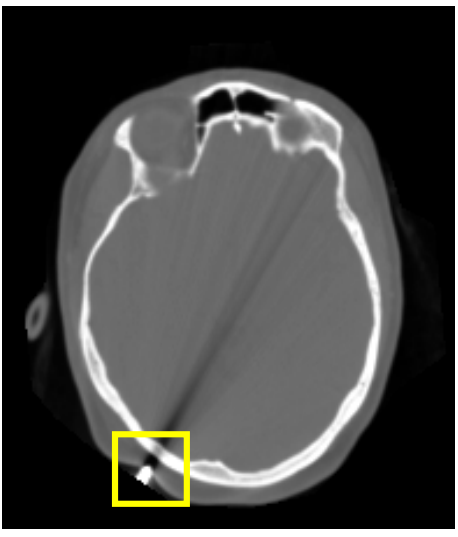

(b)

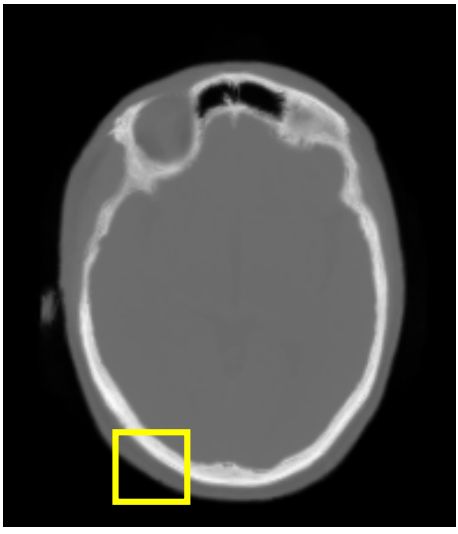

(c)

Figure 7: (a) MR, (b) atlas CT and (c) pseudo-CT for the same axial slice of patient 1. A screw in the stereotactic frame causes signal loss in the MR and streak artifacts in the CT. The pseudo-CT is mostly unaffected. If desired, the fiducial markers in the MR could be used to reintroduce the stereotactic frame in the pseudo-CT.

found that selecting a custom subset of atlas patients improved segmentations compared to a random selection. Additionally, it was found that meta-information available at the time of scanning, such as gender, age and clinical status, were as useful selection criteria as image similarity based on normalized mutual information.

A concluding remark is that CT is just a proxy, albeit a useful one, for what we really want to measure - the chemical composition. Considering the ability of MR to probe soft tissue properties it is conceivable that a more complete description could be obtained by combining the information from (pseudo-)CT and MR. This would have implications for instance for particle therapy, where the beam range is intimately tied to the chemical composition.

\section{Conclusions}

We have shown that a realistic, patient-specific, pseudo-CT of the head can be derived from an anatomical MR. This is useful for calculations on radiation transport, which are of crucial importance for both radiotherapy planning and attenuation correction of PET images.

The method used is similar to atlas-based segmentation, but, since the output is a continuous range of values instead of a segmentation, we refer to it as atlas-based regression.

We proposed a novel method of fusion, wherein the collection of deformed CTs is iteratively registered to their joint mean, and found that the resulting mean CT became more similar to the target CT. However, a significantly better model was achieved simply by taking the voxelwise median on the original set of deformed CTs. 
To visualize the resulting pseudo-CTs, we adapted an error measure - the $\gamma$-indexcommonly used to compare dose distributions when performing quality assurance in radiotherapy. The advantage of this measure is that it is robust to small displacements of high contrast objects, such as bone, which is reasonable considering the intended applications.

Atlas-based regression produced results at least as good as in earlier work, but without the need for any special imaging techniques, making it a good candidate for clinical use.

\section{Acknowledgments}

The authors would like to thank the reviewers for their valuable comments and suggestions to improve the quality of the paper. The research was supported by the Swedish Research Council (grants 2012-4281 and 2011-5176) and the Linneaus center CADICS. The work is part of the Swedish testbed for innovative radiotherapy

\section{References}

Aljabar, P., Heckemann, R. A., Hammers, A., Hajnal, J. V. \& Rueckert, D. (2009). Multi-atlas based segmentation of brain images: atlas selection and its effect on accuracy, Neuroimage 46(3): 726738.

Arsigny, V., Commowick, O., Pennec, X. \& Ayache, N. (2006). A log-Euclidean framework for statistics on diffeomorphisms, Medical Image Computing and Computer-Assisted Intervention-MICCAI 2006, Springer, pp. 924-931.

Artaechevarria, X., Munoz-Barrutia, A. \& Ortiz-de-Solorzano, C. (2009). Combination strategies in multi-atlas image segmentation: Application to brain MR data, IEEE Trans. Med. Imag. 28(8): 1266-1277.

Bezrukov, I., Mantlik, F., Schmidt, H., Schölkopf, B. \& Pichler, B. J. (2013). MR-based PET attenuation correction for PET/MR imaging, Seminars in nuclear medicine, Vol. 43, Elsevier, pp. $45-59$.

Burgos, N., Cardoso, M. J., Modat, M., Pedemonte, S., Dickson, J., Barnes, A., Duncan, J. S., Atkinson, D., Arridge, S. R., Hutton, B. F. et al. (2013). Attenuation correction synthesis for hybrid PETMR scanners, Medical Image Computing and Computer-Assisted Intervention-MICCAI 2013, Springer, pp. 147-154.

Dowling, J. A., Lambert, J., Parker, J., Salvado, O., Fripp, J., Capp, A., Wratten, C., Denham, J. W. \& Greer, P. B. (2012). An atlas-based electron density mapping method for magnetic resonance imaging (MRI)-alone treatment planning and adaptive MRI-based prostate radiation therapy, International Journal of Radiation Oncology* Biology* Physics 83(1): e5-e11.

Forsberg, D., Eklund, A., Andersson, M. \& Knutsson, H. (2011). Phase-based non-rigid 3D image registration: From minutes to seconds using CUDA, Joint MICCAI Workshop on High Performance and Distributed Computing for Medical Imaging.

Heckemann, R. A., Hajnal, J. V., Aljabar, P., Rueckert, D. \& Hammers, A. (2006). Automatic anatomical brain MRI segmentation combining label propagation and decision fusion, NeuroImage 33(1): 115-126.

Higham, N. J. (2005). The scaling and squaring method for the matrix exponential revisited, SIAM Journal On Matrix Analysis and Applications 26(4): 1179-1193.

Hofmann, M., Steinke, F., Scheel, V., Charpiat, G., Farquhar, J., Aschoff, P., Brady, M., Schölkopf, B. \& Pichler, B. J. (2008). MRI-based attenuation correction for PET/MRI: a novel approach 
combining pattern recognition and atlas registration, Journal of Nuclear Medicine 49(11): 18751883.

Hsu, S.-H., Cao, Y., Huang, K., Feng, M. \& Balter, J. M. (2013). Investigation of a method for generating synthetic CT models from MRI scans of the head and neck for radiation therapy, Phys. Med. Biol. 58(23): 8419-8436.

Janssens, G., Jacques, L., de Xivry, J. O., Geets, X. \& Macq, B. (2011). Diffeomorphic registration of images with variable contrast enhancement, Journal of Biomedical Imaging 2011: 3.

Johansson, A., Garpebring, A., Karlsson, M., Asklund, T. \& Nyholm, T. (2013). Improved quality of computed tomography substitute derived from magnetic resonance (MR) data by incorporation of spatial information-potential application for MR-only radiotherapy and attenuation correction in positron emission tomography, Acta Oncologica 52 (7): 1369-1373.

Johansson, A., Karlsson, M. \& Nyholm, T. (2011). CT substitute derived from MRI sequences with ultrashort echo time, Med. Phys. 38(5): 2708-2714.

Jonsson, J. H., Karlsson, M. G., Karlsson, M. \& Nyholm, T. (2010). Treatment planning using MRI data: an analysis of the dose calculation accuracy for different treatment regions, Radiation Oncology 5: 62 .

Kapanen, M. \& Tenhunen, M. (2013). T1/T2*-weighted MRI provides clinically relevant pseudo-CT density data for the pelvic bones in MRI-only based radiotherapy treatment planning, Acta Oncologica 52(3): 612-618.

Knutsson, H. (1989). Representing local structure using tensors, Proc. 6th Scandinavian Conf. on Image Analysis, Vol. 1019, pp. 244-251.

Knutsson, H. \& Andersson, M. (2003). What's so good about quadrature filters?, IEEE Int. Conf. Image Processing, 2003, Vol. 3, pp. III-61.

Knutsson, H. \& Andersson, M. (2005). Morphons: segmentation using elastic canvas and paint on priors, IEEE Int. Conf. Image Processing, Vol. 2, pp. II-1226.

Low, D. A., Harms, W. B., Mutic, S. \& Purdy, J. A. (1998). A technique for the quantitative evaluation of dose distributions, Med. Phys. 25(5): 656-661.

Otsu, N. (1979). A threshold selection method from gray-level histograms, IEEE Trans. Syst., Man, Cybern. 9(1): 62-66.

Persoon, L. C., Podesta, M., van Elmpt, W. J., Nijsten, S. M. \& Verhaegen, F. (2011). A fast three-dimensional gamma evaluation using a GPU utilizing texture memory for on-the-fly interpolations, Med. Phys. 38(7): 4032-4035.

Pluim, J. P., Maintz, J. A. \& Viergever, M. A. (2003). Mutual-information-based registration of medical images: a survey, IEEE Trans. Med. Imag. 22(8): 986-1004.

Plumat, J., Andersson, M., Janssens, G., Orban de Xivry, J., Knutsson, H. \& Macq, B. (2009). Image registration using the Morphon algorithm: an ITK implementation, Insight Journal . http://hdl.handle.net/1926/1527.

Raaymakers, B., Lagendijk, J., Overweg, J., Kok, J., Raaijmakers, A., Kerkhof, E., van der Put, R., Meijsing, I., Crijns, S., Benedosso, F. et al. (2009). Integrating a 1.5 T MRI scanner with a 6 MV accelerator: proof of concept, Phys. Med. Biol. 54(12): N229-N237.

Rasch, C., Barillot, I., Remeijer, P., Touw, A., van Herk, M. \& Lebesque, J. V. (1999). Definition of the prostate in CT and MRI: a multi-observer study, International Journal of Radiation Oncology* Biology* Physics 43(1): 57-66.

Rohlfing, T., Russakoff, D. B. \& Maurer Jr, C. R. (2004). Performance-based classifier combination in atlas-based image segmentation using expectation-maximization parameter estimation, IEEE Trans. Med. Imag. 23(8): 983-994.

Schreibmann, E., Nye, J. A., Schuster, D. M., Martin, D. R., Votaw, J. \& Fox, T. (2010). MRbased attenuation correction for hybrid PET-MR brain imaging systems using deformable image registration, Med. Phys. 37(5): 2101-2109.

Sjölund, J., Eriksson Järliden, A., Andersson, M., Knutsson, H. \& Nordström, H. (2014). Skull segmentation in MRI by a support vector machine combining local and global features, IEEE 
22nd Int. Conf. on Pattern Recognition. In press.

Sled, J. G., Zijdenbos, A. P. \& Evans, A. C. (1998). A nonparametric method for automatic correction of intensity nonuniformity in MRI data, IEEE Trans. Med. Imag. 17(1): 87-97.

Stanescu, T., Jans, H., Pervez, N., Stavrev, P. \& Fallone, B. (2008). A study on the magnetic resonance imaging (MRI)-based radiation treatment planning of intracranial lesions, Phys. Med. Biol. 53(13): 3579-3593.

Thirion, J.-P. (1998). Image matching as a diffusion process: an analogy with Maxwell's demons, Medical image analysis 2(3): 243-260.

Uh, J., Merchant, T. E., Li, Y., Li, X. \& Hua, C. (2014). MRI-based treatment planning with pseudo CT generated through atlas registration, Med. Phys. 41(5): 051711.

Viola, P. \& Wells III, W. M. (1997). Alignment by maximization of mutual information, International journal of computer vision 24(2): 137-154. 